\title{
Influence of Structural Parameters of Electromagnetic Harmonic Movable Teeth Transmission on System Loss
}

\author{
Yubo Ren ${ }^{*}$, Lizhong Xu and Yongli Liang \\ Parallel Robot and Electromechanical System Laboratory, Yanshan University, Qinghuangdao, Hebei, 066004, China
}

\begin{abstract}
In view of the characteristics of electromagnetic harmonic movable teeth transmission system, the heat loss has been analyzed in this paper based on basic assumptions. At the same time, the calculation methods of electromagnetic loss and mechanical loss in system have been also discussed. Moreover, the influence of changes of system parameters on electromagnetic loss and mechanical loss has been analyzed through employing the field-circuit coupling finite element method. According to the analysis results, the numerical calculation model of the three-dimensional temperature field of system can be established. And the theoretical basis can be provided for the optimization design of electromagnetic harmonic movable teeth transmission system and the improvement of transmission efficiency of transmission system.
\end{abstract}

Keywords: Electromagnetic harmonics, electromagnetic loss, mechanical loss, structural parameters.

\section{INTRODUCTION}

As electronic and control technology continues to penetrate the mechanical field, the generalized complex mechanical transmission has become a leading international subject in mechanical fields [1-4] for this concept is antitradition and could achieve the organic combination of mechanics, electricity and control. The electromagnetic harmonic movable tooth transmission system involved in this paper is a kind of organic combination of harmonic transmission technology, electromagnetic transmission technology, movable tooth transmission technology and control technology, that is, a new electromechanical integrated complex transmission system $[5,6]$.

The electromagnetic harmonic movable tooth transmission motor is an electromagnetic -mechanical energy conversion device. Due to its long-term continuous operation, energy loss is an essential part of the operation. The lost energy has a great influence on the electromagnetic harmonic movable tooth transmission motor and its structure design. Such as, the lost energy is eventually converted to heat, so that the temperature of each part of the motor increases which has a direct impact on the life of all the insulating materials. Meanwhile, the viscosity of lubricating oil of the motor may decrease, which may damage the lubrication state between the movable tooth, center wheel and tooth holder, and ultimately limit the output of the system. And the electromagnetic harmonic transmission is operating at a low speed, its running state corresponds to the operating state of starting or braking and the efficiency distribution has a great influence on the electromagnetic motor and its design and structure.

Therefore, the study of the losses of electromagnetic harmonic movable teeth transmission is of great significance

\footnotetext{
*Address correspondence to this author at the Parallel Robot and Electromechanical System Laboratory, Yanshan University, Qinghuangdao, Hebei, 066004, China; Tel: +8613833595616; E-mail: ryb@ysu.edu.cn
}

to analyze the failure mechanism of electromagnetic harmonic movable teeth transmission system and improve the operating performance as well as effectively control the lubricating and the cooling. Experts and scholars have conducted extensive researches on motor losses. For example, in literature [7], the motor losses of sine-wave drive and square-wave drive have been analyzed and it was found that the motor losses would be minimized when the optimal leading angle is controlled; in literature [8], the importance of space harmonic magnetic field on rotor losses has been revealed and the concept of harmonic losses has been proposed; at the same time, the motor stator loss, the rotor copper loss, the iron loss and the eddy current loss under different operating conditions have been also analyzed in detail; in literature [9], the concepts of stator and rotor regionalization have been proposed and the losses as well as the flux density of each region have been calculated; meanwhile, the variation principles of losses in each part of motor have been analyzed, which provided a basis for reducing the motor loss; in literature [10], the radial flux density and the tangential flux density in different parts of motor have been analyzed; it was proposed that the motor loss could be divided into alternating loss and rotational loss; at the same time, the influences of torque and speed on motor loss have been compared. The motor loss has been analyzed from a certain perspective in above literatures and the field-circuit coupling finite element method is rarely employed to study the electromagnetic losses; moreover, the literatures about the influence of operating parameters on system losses are less.

In view of the operating characteristics of electromagnetic harmonic movable teeth transmission and the system conditions at different frequencies, the electromagnetic losses and the mechanical losses of electromagnetic harmonic movable teeth transmission have been discussed in this paper. At the same time, the influence principles of structural parameters on electromagnetic losses and mechanical losses have been analyzed through taking the 
prototype as example, which provides a theoretical basis for the optimal design of system structure.

\section{LOSS ANALYSIS}

Electromagnetic harmonic tooth transmission is an electromagnetic - mechanical energy conversion device. Energy loss in action is eventually converted to heat energy and it finally reaches steady state equilibrium by the heat exchange between the motor components with the environment. The loss of the electromagnetic harmonic tooth transmission is comprised of electromagnetic loss and mechanical loss.

\subsection{Electromagnetic Loss}

Part of system input electric power is consumed in the stator resistance, producing the electron copper loss, while the other part of the consumption is in the resistance field, resulting in the stator iron loss. Besides, the additional loss including flexspline eddy current loss and hysteresis loss should also be considered. The remaining power is applied to flexspline through the air gap magnetic field- $P_{M}$.

\subsubsection{Electronic Copper Loss}

In the harmonic electromagnetic tooth transmission process, the alternating electric current flows through a conductor which will lead to the skin effect and proximity effect in the surrounding magnetic field [11]. The additional losses caused by them are defined as eddy-current loss, while the eddy-current loss and DC loss are collectively known as AC loss (copper loss) [12], namely

$P_{c u}=P_{z}+P_{w}$

$P_{c u}$ is the AC loss; $P_{z}$ is the DC loss; $P_{z}=I^{2} R_{z} ; P_{w}$ is eddycurrent loss; $I$ is the virtual value of current; $R_{z}$ is $\mathrm{DC}$ resistance.

Assuming that the magnetic field in the harmonic motor slot is parallel to its bottom, ignore the effects of eddy current of the conductor on the magnetic field and the eddycurrent loss of the conductor is shown as follows [13]:

$P_{w}=\frac{\pi \omega^{2} B^{2} l_{d} d^{4}}{128 \rho_{c}}$

$d$ is the diameter of the conductor; $l_{d}$ is the length of the conductor; $\rho_{c}$ is the resistivity of the conductor; $B$ is the amplitude of flux density; $\omega$ is the frequency of flux density angular.

\subsubsection{Iron Core Loss}

The stator core loss is one of the main losses of harmonic electromagnetic tooth transmission system, which accounts for a large proportion of the total losses. It is usually refered to the sum of tooth iron loss and iron loss of the yoke part.

$P_{F e}=P_{c}+P_{a}$

and

$$
\left.\begin{array}{l}
P_{c}=k_{z} p_{0} B_{c}^{2} f^{13} G_{c} \\
P_{a}=k_{a} p_{0} B_{a}^{2} f^{13} G_{a}
\end{array}\right\}
$$

$k_{z}, k_{a}$ is the coefficient with additional loss; $p_{0}$ is the specific losses of core material; $B_{c}$ is the tooth flux density; $B_{a}$ is the yoke flux density; $f$ means the frequency; $G_{c}$ means the tooth weight; $G_{a}$ means the yoke weight.

\subsection{Mechanical Power Loss}

\subsubsection{The Power Loss of Engagement Pair}

The analysis indicates that there is relative rolling motion between the movable tooth and flexible wheel, there is relative sliding between the movable tooth and the tooth carrier, and there are both relative rolling and relative sliding between the tooth and the center wheel $P_{1}^{i}, P_{2}^{i}, P_{3}^{i}$ respectively refering to the power loss when the $i^{\text {th }}$ movable tooth contacts with the flexible wheel, the tooth carrier and the center wheel. The meshing power loss of a single tooth is:

$$
\begin{aligned}
& P_{f}^{i}=P_{1}^{i}+P_{2}^{i}+P_{3}^{i} \\
& P f=\sum_{i=m}^{n} P_{f}^{i}+\sum_{i=p}^{q} P_{f}^{i} \\
& f^{\prime}
\end{aligned}
$$

in the formula, $F_{1 i f}^{\prime}, F_{2 i f}^{\prime}, F_{3 i f}^{\prime}$ means the friction between the movable tooth and the flexible wheel, tooth rack and center wheel; $f_{1 i}$ and $f_{2 i}$ is the friction coefficient of the movable tooth with the flexible wheel and tooth rack; $\omega_{1 i}$ means the relative rolling angular velocity between the movable tooth and the flexible wheel; $V_{2 i}$ means the relative sliding angular velocity between the movable tooth and the tooth rack; $\omega_{3 i}$ means the relative rolling-sliding angular velocity; $f_{3 i}$ means the rolling-sliding friction coefficient between the movable tooth and the flexible wheel.

The electromagnetic harmonic movable tooth transmission has multi-tooth engagement simultaneously; at any time, the friction power loss on each movable tooth is not the same, coupled with the input power, which is because there are always half of the movable teeth in two regions of engagement operating, so the power loss at any moment is shown as follows:

$$
P_{f}=\sum_{i=m}^{n} P_{f}^{i}+\sum_{i=p}^{q} P_{f}^{i}
$$

$m, n$ and $p, q$ respectively represents the number of movable tooth in two different regions of engagement.

\subsubsection{The Power Loss of the Bearing}

The power loss of a single bearing 


$$
P_{z c}=\pi n_{z} M_{f} / 30
$$

$n_{z}$ is the rotation speed of the inner ring of the bearing; $M_{f}$ is the friction torque of the bearing.

$$
M_{f}=\left\{\begin{array}{cl}
2 Y f_{1} F_{a} D_{m}+10^{3} f_{0}\left(v n_{z}\right)^{\frac{2}{3}} D_{m}^{3} & v n_{z} \geq 2 \times 10^{-3} \\
2 Y f_{1} F_{a} D_{m}+16 f_{0} D_{m}^{3} & v n_{z} \geq 2 \times 10^{-3}
\end{array}\right.
$$

$Y$ and $F_{a}$ respectively represents the axial coefficient and axial load of the bearing; $v$ is the kinematic viscosity of the lubricant; $D_{m}$ is the average diameter of the bearing; $f_{0}$ and $f_{1}$ are the coefficients related to the bearing type and lubrication methods.

\subsubsection{Power Loss Due to Oil Agitation}

The power loss due to oil agitation mainly includes that of flexspline and movable tooth. For the spherical surface (radius of $r^{\prime}$ and the effective area $A$ ) rotating at the angular velocity of $\omega^{\prime}$ in the viscous fluid medium (density $\rho^{\prime}$, kinematic viscosity $v$ and friction coefficient $f^{\prime}$ ), the power loss due to oil agitation is shown as follows:

$P_{y}=\frac{1}{8} \rho^{\prime} f^{\prime} A \omega^{\prime 3} r^{3}$

In the above formula

$f^{\prime}=\left\{\begin{array}{cc}16 / R_{e} & T_{a}<41 \\ 3\left(R_{e} / 2500\right)^{0.856}\left(16 / R_{e}\right) & R_{e} \geq 2500 \\ 1.3\left(T_{a} / 41\right)^{0.539}\left(16 / R_{e}\right) & T_{a} \geq 41\end{array}\right.$

The Reynolds number $R_{e}=\omega^{\prime} r C / v$, the Taylor number $T_{a}=R_{e} \sqrt{C / r^{\prime}}, C$ is the characteristic gap surrounding the ball surface.

The total losses of mechanical power is shown as follows:

$P=P_{f}+P_{z}+P_{y}$

\subsubsection{Rolling-Sliding Friction Coefficient}

Based on the previous analysis, there are both rolling and sliding when the tooth contacts with the center wheel, so the corresponding friction is referred to as rolling-sliding friction. The work done by the rolling friction, sliding friction and rolling-sliding friction, is respectively shown as follows:

$$
\begin{aligned}
& w_{1}=\mu_{1} \cdot F_{3 i} \cdot S_{1} \\
& w_{2}=\mu_{2} \cdot F_{3 i} \cdot S_{2} \\
& w_{3}=f_{3 i} \cdot F_{3 i} \cdot S_{3}
\end{aligned}
$$

$\mu_{1}$ refers to the rolling friction coefficient; $\mu_{2}$ is the sliding friction coefficient; $f_{3 i}$ is the rolling-sliding friction coefficient; $F_{3 i}$ is the positive pressure imposed on the movable tooth by the center wheel; $S_{1}$ refers to the arc length of engagement of movable tooth, that is, the rolling distance; $S_{2}$ refers to the sliding distance; $S_{3}$ refers to the arc length of engagement of center wheel, that is, the rolling-sliding distance.

The work done by the rolling-sliding friction is equal to the sum of the work done by rolling friction and sliding friction; therefore

$f_{3 i} \cdot S_{3}=\mu_{1} \cdot S_{1}+\mu_{2} \cdot S_{2}$

$S_{1}$ can be approximately calculated by the following formula: $S_{1} \approx r_{b} \varphi_{v}$

$r_{b}$ is the radius of the movable tooth; $\varphi_{v}$ is the wrap angle of the centered tooth; $z_{z}$ is the wave number of the centered tooth.

$S_{3}=\left(r+2 r_{b}\right) \varphi_{2}$

$r$ is the radius of flexspline.

$S_{3}=S_{1}+S_{2}$

Finally

$f_{3 i}=\left[\left(\mu_{1}-\mu_{2}\right) r_{b} \varphi_{v}+\mu_{2} \varphi_{2}\left(r+2 r_{b}\right)\right] /\left(r+2 r_{b}\right) \varphi_{2}$

\section{INFLUENCE OF SYSTEM PARAMETERS ON ELECTROMAGNETIC LOSS}

In this part, the influence of system parameters on electromagnetic loss has been analyzed through taking twopole 24-slot harmonic transmission as example. The main parameters of system are shown in Table $\mathbf{1}$.

Table 1. Harmonic electromagnetic movable teeth transmission motor parameters.

\begin{tabular}{|c|c|c|c|}
\hline Item & Value & Item & Value \\
\hline \hline Rated power $P_{e}$ & $100 \mathrm{~W}$ & Rated voltage $U_{e}$ & $220 \mathrm{~V}$ \\
\hline Number of pole pairs $p$ & 1 & Stator slots $m$ & 24 \\
\hline Number of turns per slot $N_{z}$ & 81 & Rated current $I$ & $0.56 \mathrm{~A}$ \\
\hline Outer diameter of stator $\mathrm{D}(\mathrm{mm})$ & 118 & Flexspline length $l(\mathrm{~mm})$ & 68 \\
\hline Length of iron core $l \mathrm{~T}(\mathrm{~mm})$ & 63 & Flexspline thickness $t(\mathrm{~mm})$ & 127 \\
\hline Outer diameter of flexspline $\mathrm{r}(\mathrm{mm})$ & 67 & Core material of stator & 0.2 \\
\hline air gap $\delta(\mathrm{mm})$ & 0.5 & Phase inductance $L$ & $35 \mathrm{WW} 270$ \\
\hline Phase Resistance $R(\Omega)$ & 0.188 & $0.234 \mathrm{mH}$ & \\
\hline
\end{tabular}




\subsection{Influence of Frequency on Iron Core Loss}

In order to save energy and reduce stator losses, the most direct way is to use the low-loss iron core materials. The stator core adopted in this paper uses the ultra-thin, low-loss and cold-rolled silicon steel. The specific loss curve of coldrolled non-oriented silicon steel of $35 \mathrm{ww} 270$ under different frequencies which is measured by $\mathrm{AC}$ magnetic gauge as shown in Fig. (1). It is required to employ the specific loss curve under each frequency, analyze the software SAP and adopt the best estimation method to fit the loss coefficients. Then, the relationship between core loss coefficient and frequency under alternating magnetic condition can be obtained, which is shown in Fig. (2).

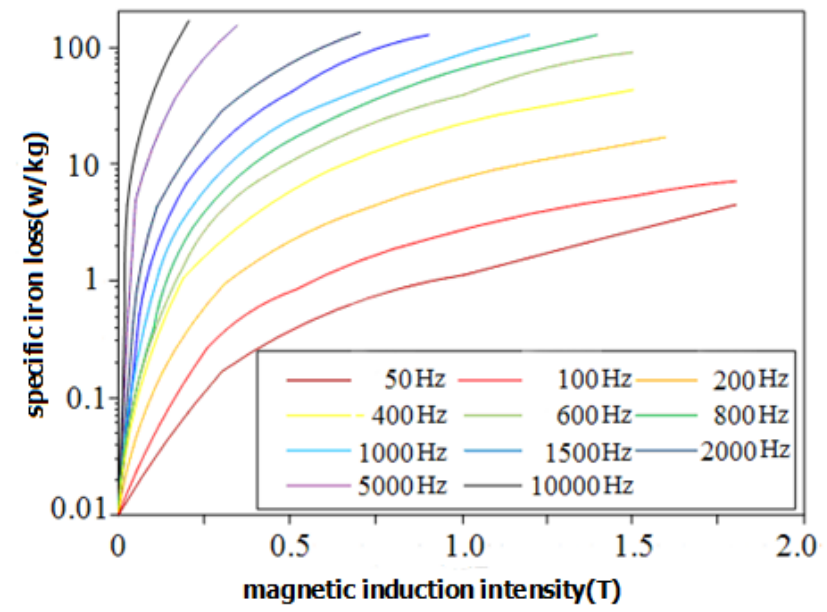

Fig. (1). The specific core loss of 35WW270 silicon steel sheet with different frequencies.

In Fig. (2), it is found that the loss coefficient of silicon is not a constant, but a function of magnetic frequency. As the frequency of motor system studied in this paper is low, the relationship between loss coefficient and frequency under low and intermediate frequencies is only analyzed. Under the low frequency (less than $400 \mathrm{~Hz}$ ) and the intermediate frequency (from $400 \mathrm{~Hz}$ to $1000 \mathrm{~Hz}$ ), the loss coefficients $k_{z}$ and $k_{a}$ have little changes with the changes of frequency. The loss coefficient can be considered as a constant when calculating the losses, which can be obtained by the loss curve fitting provided by manufacturers.

\subsection{Influence of the Waveforms of Magnetic Field on Iron Core Loss}

The waveform of the main magnetic field can directly affect the distribution of flux density in stator core. Therefore, the losses of stator core losses are related to the waveform of magnetic field. From the perspective of reducing the iron core losses, the influence of higher harmonics of flux density waveform has been analyzed under the existence of power in this paper.

In order to accurately calculate the core loss, firstly, it is required to employ the finite element to analyze the magnetic field of system. The sine-wave magnetic field and the third harmonic magnetic field should be inlet under related load. It is necessary to conduct a calculation when the motor magnetic field rotates 0.36 electrical angle. It is demanded to calculate the magnetic field distribution of 1000 magnetic field positions. Then, the waveforms of magnetic flux density of the central teeth and the yoke part of stator core in

(a) Loss coefficient $k_{z}$

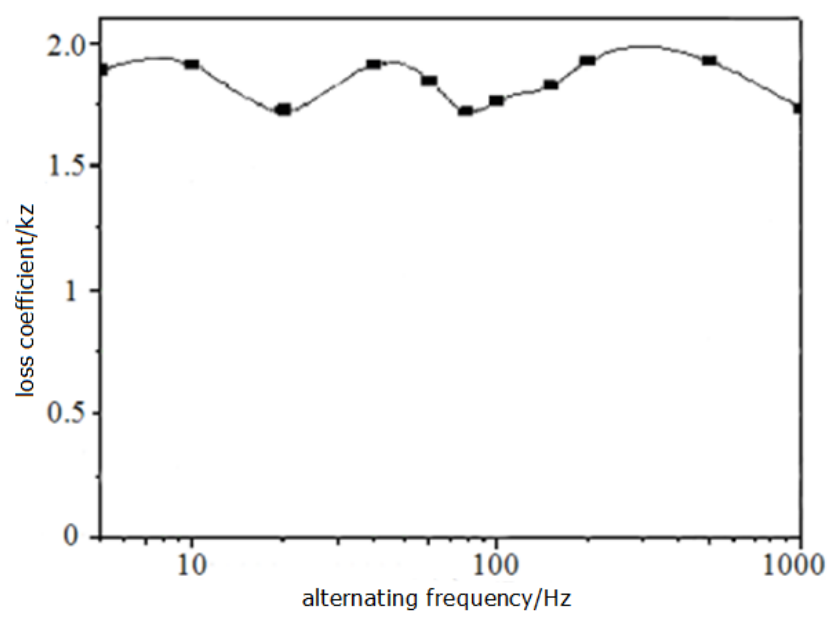

(b) Loss coefficient $k_{a}$

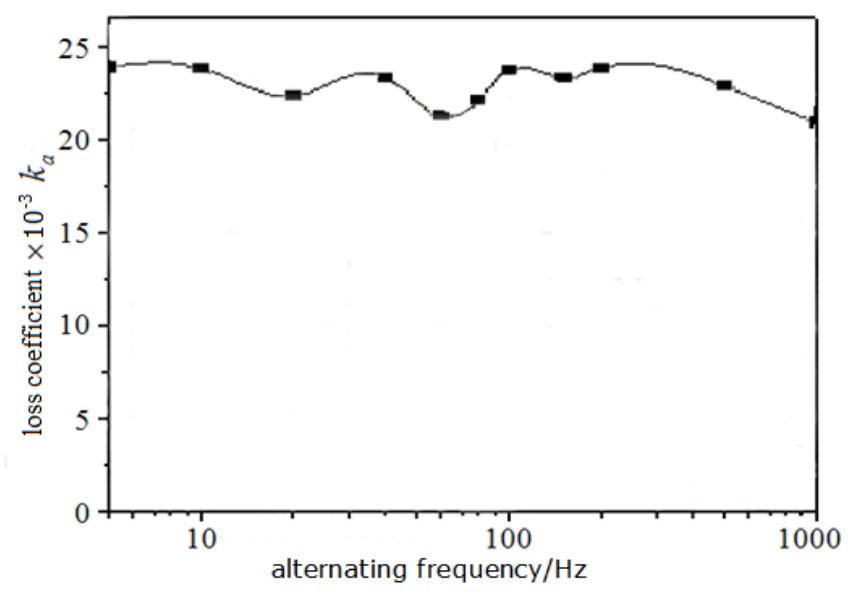

Fig. (2). The loss coefficients variation with frequency.

a cycle should be extracted, which is shown in Fig. (3). In this figure, the magnetic flux density waveforms of sinewave magnetic field are represented as long dotted line "_ _ " and the magnetic flux density waveforms of the third harmonic magnetic field are represented as continuous solid line "- ".

In Fig. (3), it is found that the magnetic flux density waveforms in teeth part and the yoke part have great changes under the sine-wave magnetic field and the third harmonic magnetic field. The contents of stator flux density harmonics under the third harmonic magnetic field are higher than the ones under the sine-wave magnetic field. Therefore, the stator core losses are also greater. For the great harmonic contents, there is a big disadvantage on the stator core loss. It is required to take measures to reduce the armature current harmonic contents so as to reduce the core losses. 


\subsection{Influence of Groove Size of Stator on Core Loss}

According to the above analysis, it is found that the stator core loss is proportional to the square of flux density and the flux density in stator groove is related to the groove size. Therefore, the stator core loss is also related to the groove size.

(a) Magnetic flux density waveforms in teeth part

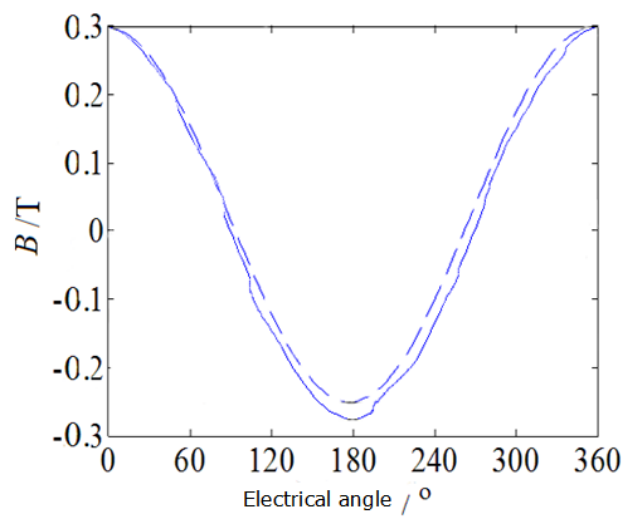

(b) Magnetic flux density waveforms in yoke part

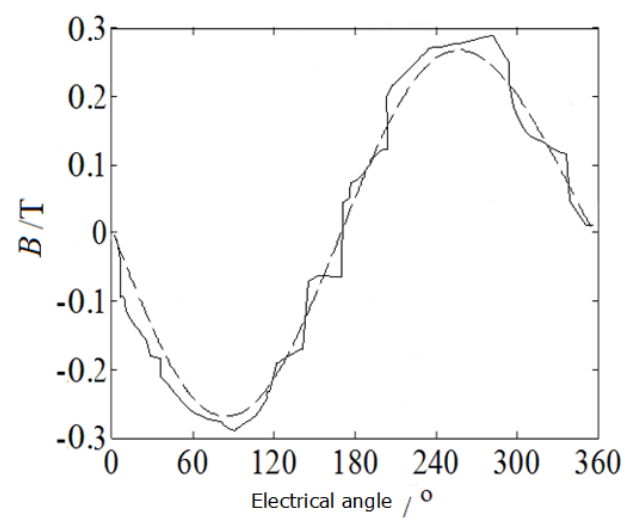

Fig. (3). Stator core flux density waveform.

In this paper, the prototype power is $0.5 \mathrm{kw}$ and the structure of stator groove as well as the size is shown in Fig. (4).

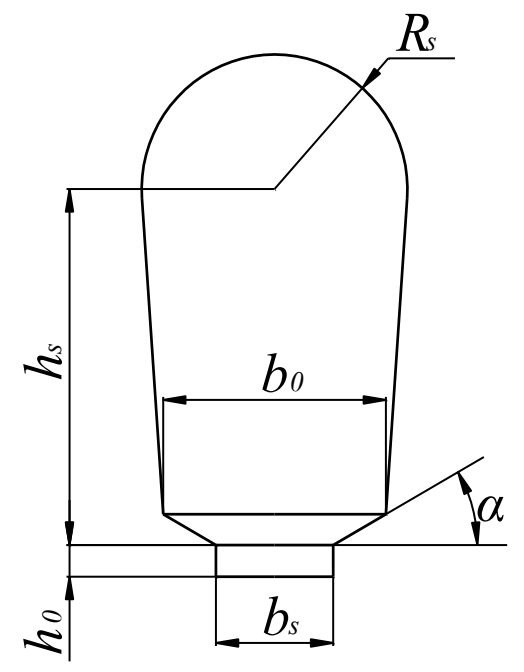

Fig. (4). Basic sizes of stator slots.
Based on the motor loss calculation model of field-circuit coupling finite element, the influences of the slot width, the slot height, the groove humeral angle, the groove width, the groove height and the radius size of stator have been analyzed, which is shown in Fig. (5).

(a) Influence of changes of slot width on loss

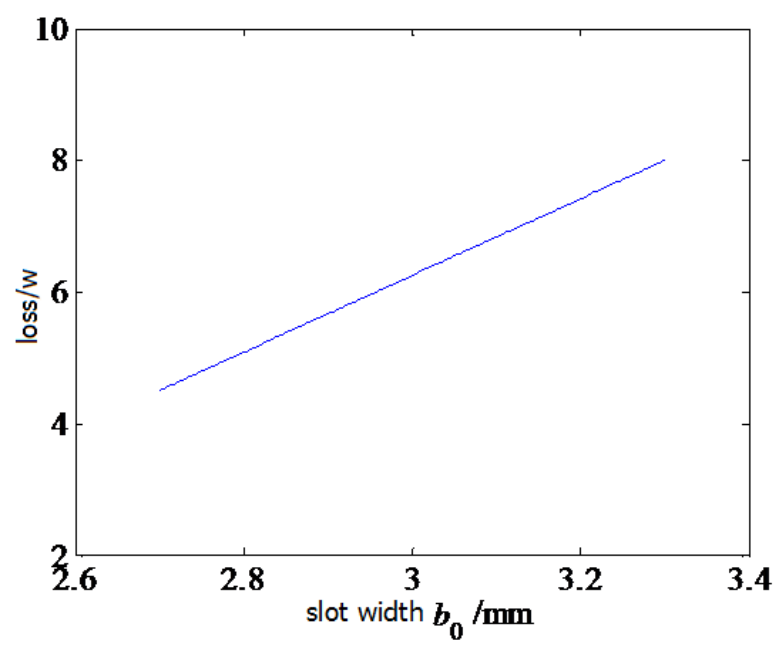

(b) Influence of changes of slot height on loss

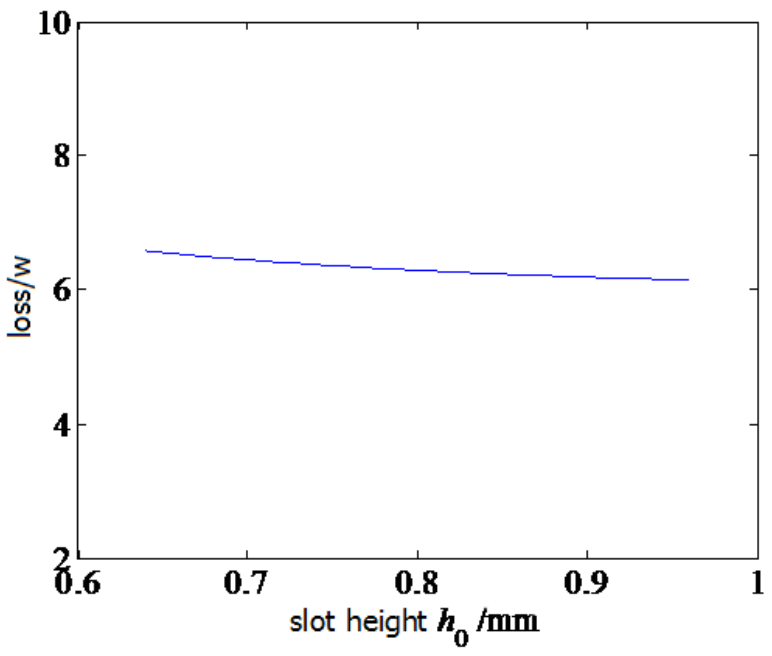

(c) Influence of groove humeral angle on loss

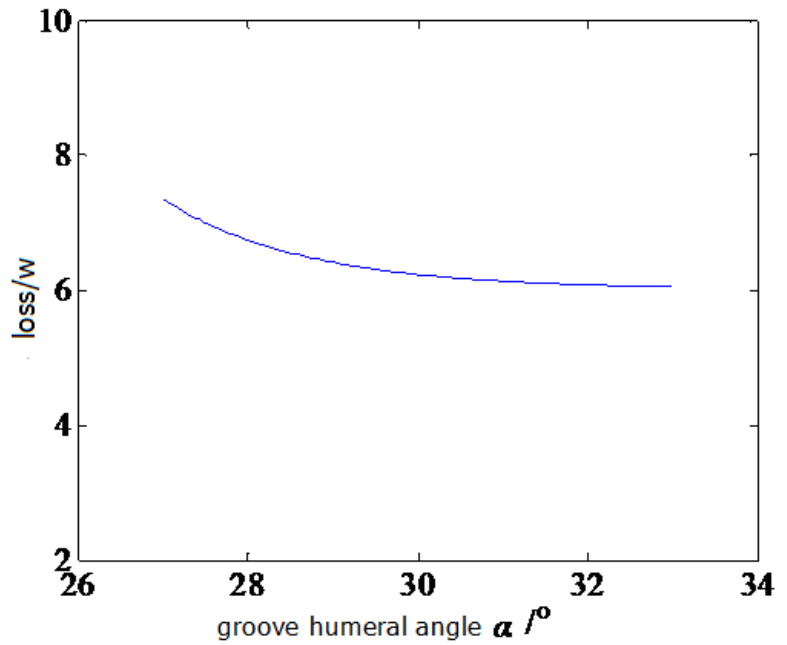


(Fig. 5) contd.....

(d) Influence of groove width on loss

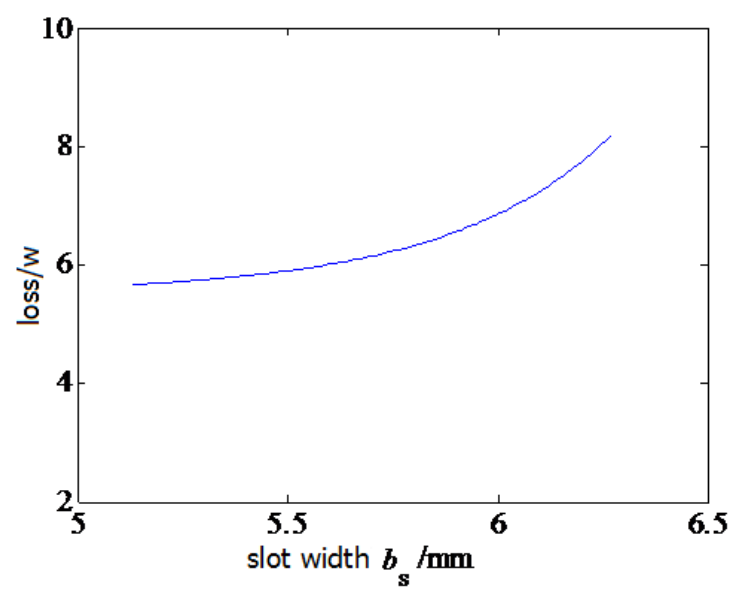

(e) Influence of groove height on loss

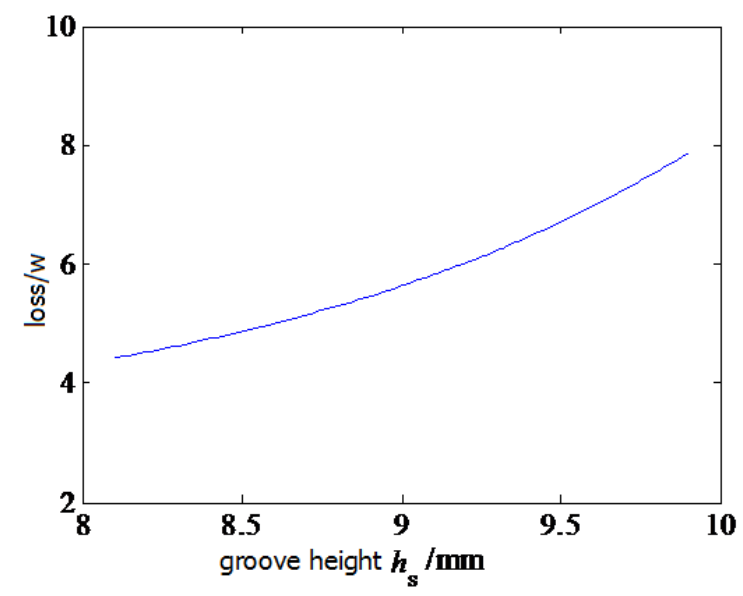

(f) Influence of groove radius on loss

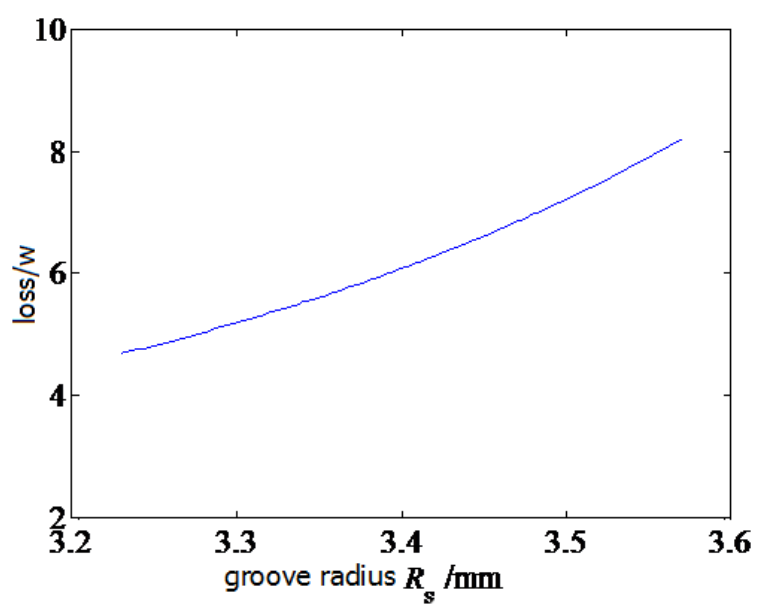

Fig. (5). Impact of different slot sizes on core losses.

In Fig. (5), it is found that:

(1) The larger the slot width is, the greater the loss will be. That is because the slotting of stator will cause the uneven gap presence and produce the presence harmonics. The presence harmonics and the magnetic potential fundamental wave can cause the presence harmonic magnetic field. The larger the slot width is, the greater the influence of harmonics on loss will be.

The influence of slot height on loss is slight due to its smaller size.

The groove humeral angle selected in this paper is among $0.9 \sim 1.1$ times $27^{\circ} \sim 33^{\circ}$ of $30^{\circ}$. The greater the groove humeral angel, the smaller the loss will be. However, the changes are gentle.

The larger the groove width is, the greater the loss will be. That is because the larger size of groove width will lead to the reduction of teeth width. When the magnetic flux of each fundamental wave is unchanged, the magnetic circuit will be more saturated and the loss will be increased.

The increase of groove height will lead to the reduction of the magnetic circuit area in yoke part. The magnetic circuit is easily saturated and the loss is increased.

(6) The increase of groove radius can cause the reduction of magnetic circuit area in yoke area. The saturation degree will be increased, which is not conducive to the reduction of loss.

However, the difference in the influence of each groove size on loss can directly affect the size of groove area. The phenomenon that the sizes which greatly affect the loss are blindly improved to reduce the loss can lead to the high groove filling rate and increase the offline difficulty. Therefore, under the premise of understanding the influence of each size on loss, it is required to reasonably select the groove size so as to reduce the losses to some extent.

\subsection{Proportional Changes of Copper Loss and Iron Loss in Electromagnetic Loss}

The electromagnetic loss in electromagnetic harmonic movable teeth transmission can be divided into electronic copper loss and iron core loss. The additional losses caused by harmonic magnetic potential and leakage magnetic field should be added into the copper loss and the iron loss through the coefficient. When the power changes from small to large, the proportional changes of the copper loss $P_{c u}$ and the iron loss $P_{F e}$ on total electromagnetic losses are shown in Fig. (6).

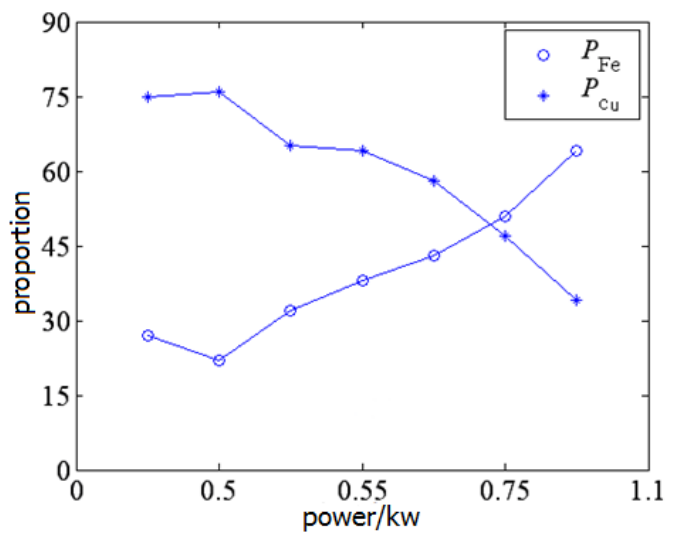

Fig. (6). Proportion of copper losses and iron losses in electromagnetic losses. 
In Fig. (6), it is found that:

(1) When the motor power changes from small to large, the proportion of copper loss is changing. The changes are from large to small which shows a declining trend;

When the motor power changes from small to large, the proportion of iron loss is changing. The changes are from small to large, which shows an upward trend; when the power is greater than $0.75 \mathrm{kw}$, the changes of copper loss are more than $50 \%$, which is greater than the ones of copper loss.

For the electromagnetic harmonic movable teeth transmission motor with small power, the proportion of copper loss in electromagnetic loss is high. It is required to take measures to control copper loss based on the factors to affect copper loss; however, for the electromagnetic harmonic movable teeth transmission motor with great power, the proportion of iron loss is greater than the one of copper loss. Therefore, it is necessary to pay attention to the flux density distribution of each part of stator so as to reduce the iron loss.

\section{INFLUENCE FACTORS AND INFLUENCE PRINCIPLES OF MECHANICAL POWER LOSS}

The mechanical power loss of electromagnetic harmonic movable teeth transmission is influenced by the teeth radius, the ratio of long shaft and short shaft of flexible gear after deformation, the transmission ratio and the lubrication effects, etc. In this paper, the change principles of the mechanical power loss affected by above factors have been analyzed and calculated. Some calculation results can be shown in Figs. (7-10)

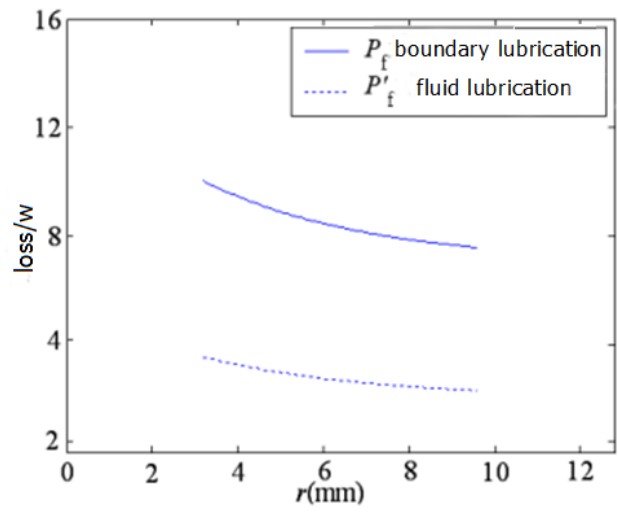

Fig. (7). Variation of Loss with Movable Tooth Radius $r_{b}$.

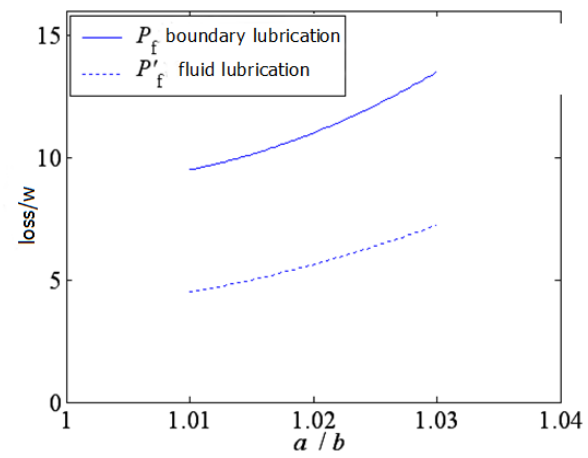

Fig. (8). Variation of Loss with $a / b$.

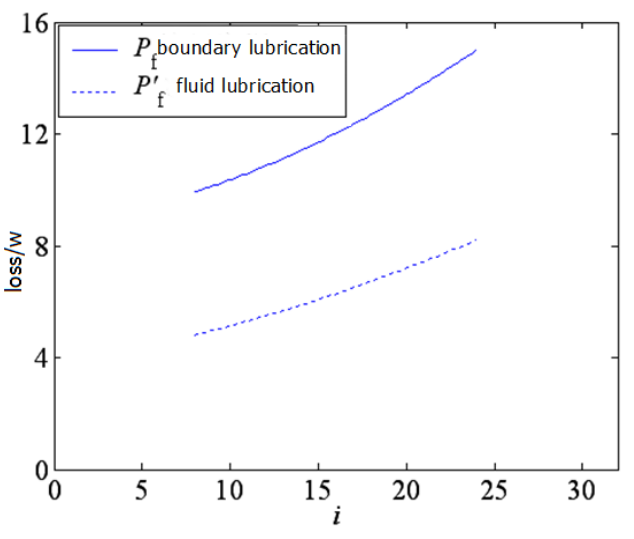

Fig. (9). Variation of loss with transmission ratio $i$.

(a) Relationship between Loss and Lubrication Condition under Different $r_{b}$.

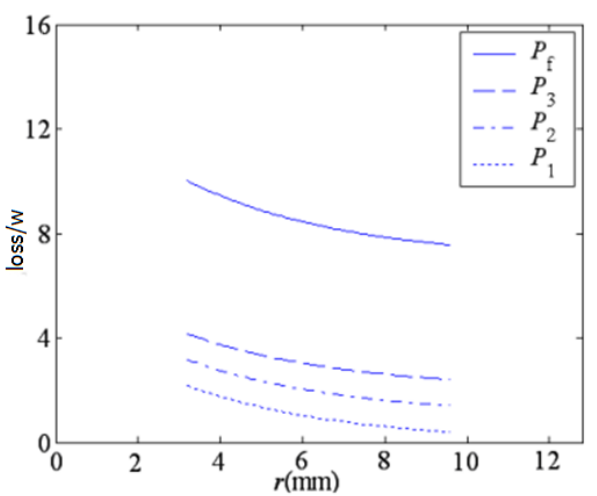

(b) Relationship between loss and lubrication condition under different $a / b$

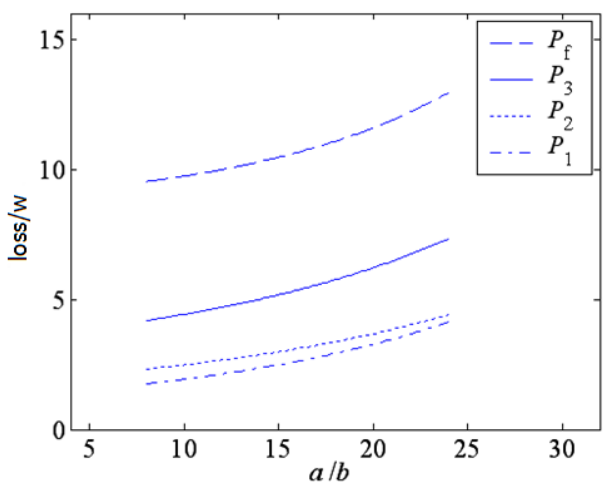

(c) Relationship between loss and lubrication condition under different $i$

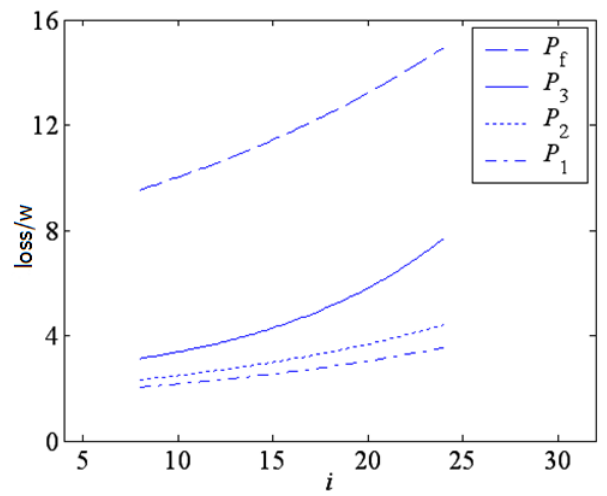

Fig. (10). Relationship curve of loss and lubrication status. 
In Figs. (7-10), it is found that:

(1) With the increase of teeth radius $r_{b}$, the friction losses between movable teeth and teeth rack and between center gear and flexible gear are both reduced. That is because the teeth radius is increased, the number of movable teeth is few and the friction pair is reduced with the certain whole size of system.

(2) With the increase of the ratio $a / b$ between long shaft and short shaft of flexible gear after deformation, the friction losses between movable teeth and teeth rack and between center gear and flexible gear are both increased. That is because the increase of $a / b$ represents the increase of radial deformation of flexible gear. If the radial deformation of flexible gear is increased, the force of flexible, center gear and teeth rack on movable teeth will be great. Therefore, the friction loss is great.

With the increase of transmission ratio $i$, the friction losses between movable teeth and teeth rack and between center gear and flexible gear are both increased. That is because the transmission ratio is increased and the number of movable teeth is increased, the friction pair will be increased. Although force on each movable tooth is reduced, the friction loss produced by the increase of friction pair can not be offset.

The influence of lubrication condition on the friction loss of electromagnetic harmonic movable teeth transmission is great. The friction loss of electromagnetic harmonic movable teeth transmission can be reduced by more than $95 \%$ through achieving the liquid lubrication. And the friction loss of harmonic movable teeth transmission can be reduced by $90 \% \sim 94 \%$ under boundary lubrication situation. The reason is that the friction coefficients between movable teeth and teeth rack and between center gear and flexible gear are low under liquid lubrication condition, which are generally between 0.001 0.008; in the boundary lubrication condition, the friction coefficients are generally between $0.03 \sim 0.1$. Therefore, the good lubrication is very important to improve the working efficiency of electromagnetic harmonic movable teeth transmission system.

The proportions of the three friction losses in overall friction losses are basically: the friction loss between movable teeth and center gear is the greatest, accounting for $40 \% \sim 50 \%$ of total friction losses, followed by the friction loss between movable teeth and teeth rack, which accounts for $30 \% \sim 50 \%$ of total friction loss; the friction loss between movable teeth and flexible gear is the smallest, accounting for $20 \% \sim 30 \%$ of total friction loss.

\section{CONCLUSION}

In this paper, the electromagnetic loss and the mechanical loss of electromagnetic harmonic movable teeth transmission have been studied and the influence principles of changes of system parameters on electromagnetic loss and mechanical loss have been analyzed. The results show that:
(1) The electromagnetic loss of system is greater than the mechanical loss.

(2) The proportion of electronic copper loss in total electromagnetic loss is high under low power. It is required to take measures to control the copper loss according to the factors that affect the copper loss.

(3) When the power is more than $0.75 \mathrm{kw}$, the proportion of iron loss in electromagnetic loss is higher than the one of copper loss. It is necessary to pay attention to the distribution of flux density in each part of stator so as to reduce the iron loss.

(4) In order to reduce the loss of mechanical power, the diameter of movable teeth and flexible gear can be determined according to the proportions of friction losses between movable teeth and center gear, between movable teeth and teeth rack and between movable teeth and flexible gear. Under the certain radial displacement, it is required to reduce the friction pair and improve the lubrication efficiency.

\section{CONFLICT OF INTEREST}

The authors confirm that this article content has no conflict of interest.

\section{ACKNOWLEDGEMENTS}

Declared none.

\section{REFERENCES}

[1] M. A. Delucchi, and M. Z. Jacobson, "Providing all global energy with wind, water, and solar power, Part II: Reliability, system and transmission costs, and policies"," Energy Policy, vol. 39, no. 3, pp. 1170-1190, 2011.

[2] N. Li, S. M. Wang, and J. J. Du, "Natural characteristics and dynamic load coefficient of power four embranchments gear transmission," Journal of Aerospace Power, vol. 28, no. 2, pp. 445451, 2013.

[3] Y. Ren, L. Xu, and Y. Wu, "Study on principle of electromechanical integrated electromagnetic harmonic oscillating teeth transmission," International Journal of Advancements in Computing Technology, vol. 4, no. 16, pp. 183-192, 2012.

[4] Y. Ren, and L. Xu, "Analysis on the finite element of electromechanic coupling of flexible gear in electromagnetic harmonic drive," Applied Mechanics and Mechanical Engineering, vol. 249-250, no. 15, pp. 771-777, 2013.

[5] Y. Ren, L. Xu, and Y. Liang, "Analysis of flexible spline deformation in electromagnetic harmonic drive motor coupling system," Journal of Yanshan University, vol. 3, no. 37, pp. 211216, 2013.

[6] Y. Ren, L. Xu, and Y. Liang, "Force and deformation analyses for flexspline of an electromechanical integrated electromagnetic harmonic friction drive," China Mechanical Engineering, vol. 22, no. 10, pp. 1169-1172, 2011

[7] J. Zou, J. Li, Y.X. Xu, and Y. Wei, "Influences of drive strategies on the ioss of permanent magnet brushless birect current motor," Transactions of China Electrotechnical Society, vol. 26, no. 9, pp. 43-47, 2011.

[8] J. Hu, Y. Luo, Z. Li, and M.-J. Liu,. "Investigtion of losses in the permanent magnet synchronous motor under different operation conditions," Electric Machines and Control, vol. 13, no. 1, pp. 1116, 2009.

[9] H. Zhao, Y. Luo, X. Liu, R.H. Wang, and W. Chen, "Analysis on no-load iron losses distribution of asynchronous motors with timestepping finite element method," In: Proceedings of the CSEE, vol. 30 , no. 30 , pp. $99-106,2010$ 
[10] L. Li, X. Huang, B. Kao, B. Kao, and B. Yan, "Research of core loss of permanent magnet synchronous motor (PMSM) in AC servo system," In: International Conference on Electrical Machines and Systenms. Piscataway, USA: IEEE, 2008, pp. 602-607.

[11] Y. Q. Wang, G. Wu, and S. Wang, Engineering Electromagnetic Fields Introduction, Electronic Industry Press, Beijing, 2005, pp. $198-210$
[12] S. Iwasaki, and R. Deodhar, "Influence of PWM on the proximity loss in permanent-magnet brushless AC machines," IEEE Transactions on Industry Applications, vol. 45, no. 4, pp. 13591367, 2009.

[13] M. Hu, and X. Huang, Method and Application of Numerical Calculation of Motor Performance, Southeast University Press, Nanjing, 2003, pp. 72-77.

(C) Ren et al.; Licensee Bentham Open.

This is an open access article licensed under the terms of the Creative Commons Attribution Non-Commercial License (http://creativecommons.org/licenses/by-nc/4.0/) which permits unrestricted, non-commercial use, distribution and reproduction in any medium, provided the work is properly cited. 